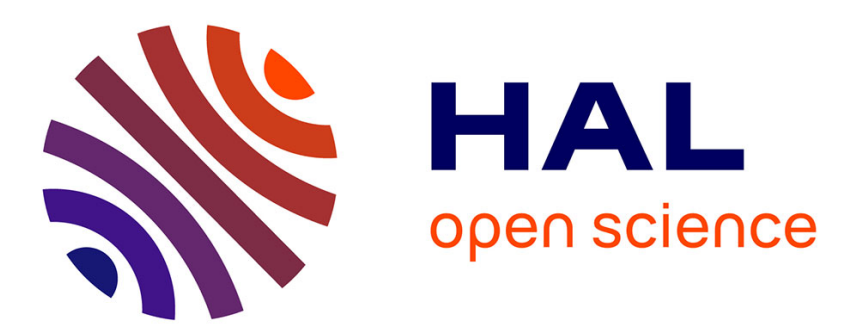

\title{
How the hydrogen sorption properties of palladium are modified through interaction with iridium
}

Christine Goyhenex, Laurent Piccolo

\section{To cite this version:}

Christine Goyhenex, Laurent Piccolo. How the hydrogen sorption properties of palladium are modified through interaction with iridium. Physical Chemistry Chemical Physics, 2017, 19 (48), pp.3245132458. 10.1039/C7CP07155H . hal-02373881

\section{HAL Id: hal-02373881 \\ https://hal.science/hal-02373881}

Submitted on 21 Nov 2019

HAL is a multi-disciplinary open access archive for the deposit and dissemination of scientific research documents, whether they are published or not. The documents may come from teaching and research institutions in France or abroad, or from public or private research centers.
L'archive ouverte pluridisciplinaire HAL, est destinée au dépôt et à la diffusion de documents scientifiques de niveau recherche, publiés ou non, émanant des établissements d'enseignement et de recherche français ou étrangers, des laboratoires publics ou privés. 


\title{
How the hydrogen sorption properties of palladium are modified through interaction with iridium
}

\author{
Christine Goyhenex, ${ }^{* a}$ and Laurent Piccolo ${ }^{b}$
}

Received Date

Accepted Date

DOI: 10.1039/xxxxxxxxxx

Hydrogen sorption (adsorption/absorption) on metals, in the form of thin films or nanoparticles, is a key process in the fields of energy storage and heterogeneous catalysis. Atomic hydrogen www.rsc.org/journalname dissolved in the subsurface of a metal affects its surface atomic and electronic structures, and thereby its surface reactivity and catalytic properties. In addition, alloy effects modify both catalytic and hydrogen sorption phenomena. In order to rationalize recent experimental results showing the negative impact of hydrogen absorption on catalysis, the present article proposes an insight into structure-reactivity relationships through computational simulations, using density functional theory, of hydrogen sorption in the near-surface region of palladium atomic layers interacting with an iridium substrate. A detailed analysis of the electronic structure using local projected densities of states (PDOS) and crystal orbital overlap population (COOP) curves was carried out. It is found that the Pd/Ir system, with respect to pure Pd surfaces, keeps acceptable adsorption properties for surface reactions while preventing hydrogen penetration. The results of electronic structure calculations show that the most important difference between $\mathrm{Pd}$ and Ir is related to the strong anti-bonding character of the $1 s-\mathrm{H} / 5 p$-Ir interaction, leading to the non-bonding character of the $s p$ Ir interaction with hydrogen. Thus, increasing the Ir concentration in a Pd-based system increases the anti-bonding contribution, which strongly weakens the overall metal-hydrogen interaction.

\section{Introduction}

Surface alloys, and subsequently nanoalloys, have received much attention because of their applications in various fields such as metallurgy, magnetism, optics and catalysis $1+3$. The mixing of two metallic elements in metallic layers or nanoalloys is expected to bring different properties from the pure materials and therefore new potential applications. In addition, by controlling metal composition and dimensionality, one can design customized materials. In the field of heterogeneous catalysis, it leads to an improvement of the performance in terms of activity, selectivity, and/or stability 2 .

From the experimental viewpoint, bimetallic supported PdIr catalysts and their monometallic counterparts were evaluated for the oxidation of $\mathrm{CO}$ and the preferential oxidation of $\mathrm{CO}$ in the presence of hydrogen (PROX). Although Pd alone is an excellent $\mathrm{CO}$ oxidation catalyst, it is poorly selective to $\mathrm{CO}_{2}$ in PROX.

\footnotetext{
a Institut de Physique et Chimie des Matériaux de Strasbourg, Université de Strasbourg, CNRS UMR 7504, 23 rue du Lœss, BP 43, F-67034 Strasbourg, France, E-Mail: christine.goyhenex@ipcms.unistra.fr

$b$ Univ Lyon, Université Claude Bernard - Lyon 1, CNRS, IRCELYON - UMR 5256, 2 Avenue Albert Einstein, F-69626 VILLEURBANNE CEDEX, France

$\dagger$ Electronic Supplementary Information (ESI) available. See DOI: 10.1039/b000000x/
}

This has been previously ascribed to the formation of Pd hydride, which would favor the (undesirable) oxidation of hydrogen 4 . Ir alone exhibits low performance for both reactions. However, the PdIr catalysts appeared much more efficient than their Ir and Pd counterparts in PROX ${ }^{5}$. This synergistic effect has been correlated to Ir-induced inhibition of hydrogen absorption in Pd through nanoalloying 6 . Indeed, the nano-alloying of Pd with elements miscible (e.g. $\mathrm{Co}, \mathrm{Ni}, \mathrm{Cu}, \mathrm{Au}, \mathrm{Ag}$ ) or immiscible (e.g. Ir, $\mathrm{Pt}, \mathrm{Rh}, \mathrm{Ru}$ ) in the bulk state leads to changes in its hydrogen absorption properties (absorption capacity, diffusion rate, hydride formation, etc.) 7.9 . In the case of Ir-Pd nanoalloys, the pressurecomposition isotherms showed a much smaller hydrogen sorption capacity as compared to Ir and Pd counterparts. Moreover, unlike Pd nanoparticles, PdIr nanoalloys do not form a $\beta$-hydride phase. Consistently with previous TEM-EDX analyzes which have suggested a surface enrichment in $\mathrm{Pd}$, computational simulations for small clusters 10 and $2 \mathrm{~nm}$-sized particles $\frac{11}{11}$ predict various chemical configurations mostly driven by a strong surface segregation of Pd and the large miscibility gap of bulk PdIr, which has profound consequences on surface reactivity $12[13$.

From the single theoretical viewpoint, the surface reactivity studies (involving hydrogen) on PdIr based systems are very scarce, unlike for pure Pd 14 and for some Pd-based bimetallics surfaces or nanoparticles 1924 . In this context, until now, the 
most advanced work on PdIr is based on DFT calculations performed on small clusters containing 13 to 79 atoms. Both structural and adsorption studies have been carried out, the latter involving benzene, hydrogen and carbon monoxide molecules 10 12 13. Although these studies show some nanosize effects both on the atomic structure and the reactivity, larger size nanoparticles are hardly handleable within first-principle calculations. A first approach to fill the gap towards larger-size systems remains to start with bimetallic surface models.

In this work, calculations of hydrogen sorption properties were performed in order to determine the physical origin of the differences between pure Pd and Pd interacting with an Ir substrate, through an accurate local analysis of the hydrogen bonding properties. Consistently with the strong surface segregation of $\mathrm{Pd}$ in PdIr systems, the calculations are made for an Ir substrate covered by an atomic overlayer of Pd which, apart from its fundamental interest, can be considered to mimic the extended facets of large core-shell nanoparticles. It is worth mentioning that one other interest of the PdIr system is the absence of strain effect, since the constituent elements present a negligible size mismatch. This enables us to explore the so-called ligand effect, coming from the heterometallic interactions between the surface layer and the substrate, independently from the strain effect. Consequently, the present results remain qualitatively valid for other couples of bulk-immiscible elements with similar lattice parameters.

\section{Methodology}

\subsection{Use of the density functional theory with the SIESTA} method

SIESTA 25 is a widely used method to perform standard selfconsistent Kohn-Sham DFT electronic structure calculations on molecules and solids. Its efficiency is tightly related to the use of localized basis sets (Numerical Atomic Orbitals: NAO). It can be used with a wide variety of exchange-correlation functionals (including the well-known and widely used GGA-PBE, as in the present work). Practical implementation of DFT calculations with SIESTA requires some particular choices including exchange correlation functional, basis set, and pseudopotentials which are used to describe the effects of the core (i.e. non-valence) electrons of an atom and its nucleus. In our case, the latter are normconserving, improved Troullier-Martins pseudopotentials 26 with core corrections, factorized in the Kleinman-Bylander form ${ }^{27}$ including scalar-relativistic effects. Pseudopotentials were generated using the program $\mathrm{ATOM}^{28}$ developed by A. Garcia et al.. Basis sets, describing the valence electrons, are numerical localized atomic-orbital sets. We used a split-valence double-zeta basis with polarization (DZP) for all the atoms of the systems. Two main relevant quantities in SIESTA are the k-grid cutoff and the mesh cutoff. The first one governs the fineness of the k-grid used for Brillouin zone sampling. The second one corresponds to the fineness of the real-space grid on which the Poisson equation is solved. In this work we took $17 \AA$ for the k-grid cutoff and 200 $R y$ for the mesh cutoff. Finally, atomic positions were systematically fully relaxed, using the conjugate gradient (CG) minimization method. The method, within these settings has been used successfully for hydrogen adsorption/absorption on/in Pd-based systems 2229 . Adsorption or absorption energy of $\mathrm{H}$, on a surface layer or in a subsurface layer of a metal $\mathrm{M}$ is evaluated from total energies relatively to the hydrogen molecule:

$$
E_{a d s, a b s}=E_{s l a b}(M H)-E_{s l a b}(M)-\frac{1}{2} E\left(H_{2}\right)
$$

where the first term corresponds to the relaxed system (Pd or $\mathrm{Pd} / \mathrm{Ir}$ slab) + an hydrogen atom at a stable adsorption or absorption site ( $M=$ metal; $H=$ hydrogen). The second term is the total energy of the relaxed bare slab. The last term corresponds to half the energy of a hydrogen molecule in the vacuum.

\section{$2.2 \mathrm{Pd} / \operatorname{Ir}(100)$ and $\operatorname{Pd} / \operatorname{Ir}(111)$ systems of layers: selected geometries for $\mathrm{H}$ sorption}

We chose to study the extended (100) and (111) model surface systems, constituted either by pure Pd and Ir slabs or by an underneath substrate of Ir further covered by a surface atomic layer of Pd. For DFT calculations, we used the supercell model including a slab and a large enough region of vacuum. These surfaces can also be considered as representative of the main facets of a large FCC nanoparticle. The slab in the supercell is infinite and periodic in the directions parallel to the surface, but finite in the direction perpendicular to the surface. The vacuum spacing should be large enough to avoid surfaces of consecutive slabs affecting each other. The calculated total energy of a 12-layers slab of Pd(111) with a $2 \mathrm{~nm}$ thick region of vacuum chosen after checking that the total energy variation is less than $1 \mathrm{meV}$ with regard to the same system with a very thick vacuum region of $4 \mathrm{~nm}$. Then, the slab itself should be thick enough to avoid any interaction between the two surfaces of one slab. This normally also ensures that the middle layer of the slab exhibits bulk-like properties and that the charge distribution at the surface is not affected by the thinness of the supercell. With 4 atoms per layer, 12 layers were considered so that calculations were still easily manageable to perform fully relaxed calculations. It is worth mentioning that the negligible atomic size mismatch between Pd and Ir ensures the correctness of simulating the Pd overlayer in total registry (pseudomorphy) with the Ir substrate. In this configuration, one atom of $\mathrm{H}$ adsorbed at the surfaces leads to a nominal coverage of $0.25 \mathrm{ML}$. In the present study, we aim at elucidating characteristics of sorption properties at low hydrogen coverage including, in particular, electronic structure features, so that further investigations as a function of hydrogen coverage would be the next step of the work.

\section{Results}

\subsection{Energetics of hydrogen sorption}

We have calculated the sorption energy of a single hydrogen atom at the different possible sites on the (111) and (100) surfaces of $\mathrm{Pd}$ and Pd/Ir systems including subsurface sites (let us recall here that in the used slab configuration, the nominal $\mathrm{H}$ coverage is $0.25 \mathrm{ML}$ ). These sites, according to the FCC materials symmetry are well defined: threefold hollow FCC and HCP, twofold bridge, top and subsurface interstitial tetrahedral or octahedral (under hollow sites) for (111) surface, hollow (four-fold), bridge, top 
and subsurface octahedral $\left(\mathrm{O}_{h}\right.$, under top sites) or tetrahedral ( $\mathrm{T}_{d}$, under bridge) sites for (100) surfaces. The geometric configurations of the systems (slabs + interacting hydrogen atom) are represented in Fig. 1 .
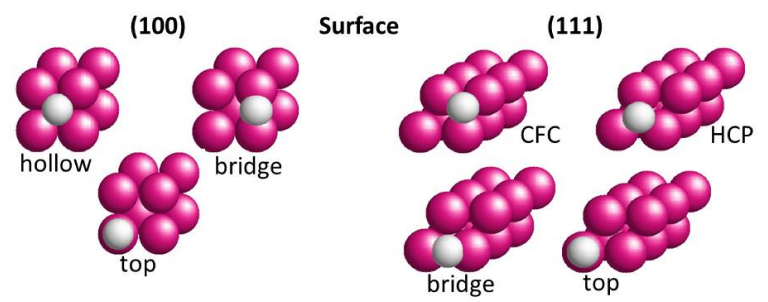

Subsurface
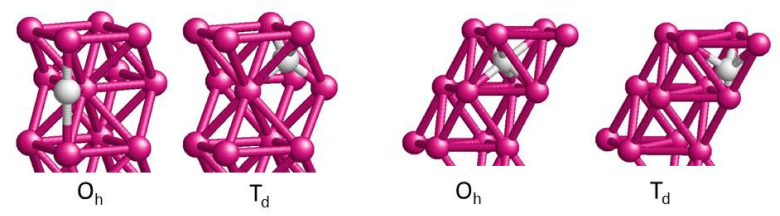

Fig. 1 Models for the geometric configurations of the system (metal slab + interacting hydrogen). The main sites for adsorption on (111) and (100) are represented in the upper images (ball representation) and the main sites for absorption in the first subsurface layer are represented in the lower images (ball and sticks representation). White ball: hydrogen atom; pink balls: metal atoms. $T_{d}$ : tetrahedral site; $O_{h}$ : octahedral site.

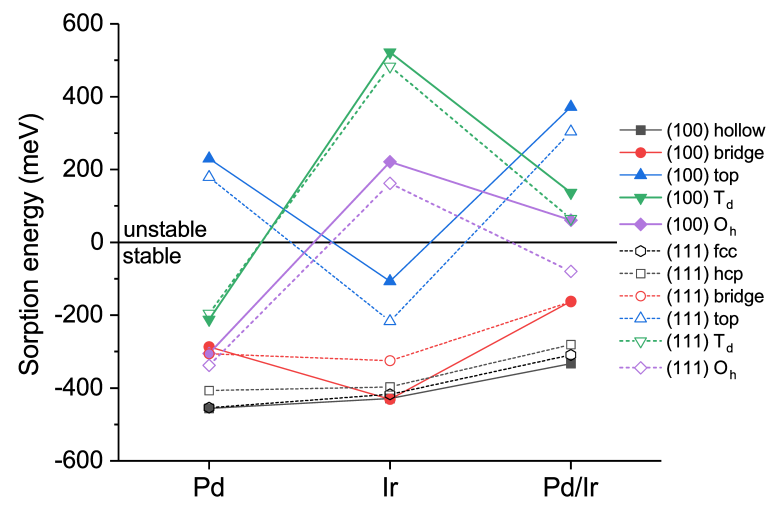

Fig. 2 Summarize of the hydrogen sorption energies for each considered system (see Fig. 11. The horizontal line at $x=0$ separates the region of unstable positions (positive energies) from the region of stable ones (negative energies). Filled symbols linked by solid lines correspond to sorption at (100) surfaces. Empty symbols linked by dashed lines correspond to sorption at (111) surfaces.

The hydrogen sorption energies, determined from DFT calculations and using eq. 1, are summarized in Fig. 2. The numerical values are given in the tables of the supplementary information section. From eq. 1, a negative adsorption energy corresponds to a stable state compared to free hydrogen molecules. The fact that hydrogen adsorption in Pd has been widely studied provides here a good verification test of the relevance of the present calculations within the SIESTA method, since the main characteristics as found in the literature are well reproduced. Briefly, on (100) surfaces, hollow sites are the most stable ones and one-fold coordinated on-top sites are very unstable (positive sign). Absorption at subsurface octahedral and tetrahedral sites is rather stable since the associated energies show a negative sign and values comparable to adsorption ones.

On (111) surfaces, we can make the same ascertainments on the relevance of our results compared to the literature. The present results for $\mathrm{H} / \mathrm{Pd}(111)$ are well consistent with previous reference studies $14 \mid 15 / 20$. The favored sites are the three-fold coordinated FCC and HCP and the least stable the one-fold coordinated top ones (positive adsorption energy in our case). Again, subsurface sites are rather stable and, with negative values of the absorption energy, are competing with adsorption sites.

One particularity was also reproduced in our study, already mentioned by Qi et al.17: upon geometry optimization, the hydrogen could stabilize its position in all tested sites except for the bridge one from which hydrogen spontaneously transfers to FCC site without diffusion energy barrier during the relaxation process (therefore the energy reported in Fig. 2 is the one obtained with $z$ optimization only). In summary, for Pd surfaces, consistently with previous works, chemisorption at surface hollow sites is the most stable but subsurface sites are also rather stable (negative sign) in agreement with the tendency to form hydrides in bulk Pd. While hydrogen chemisorption on Pd surfaces has been widely investigated, interaction of hydrogen with Ir surfaces has been the subject of only few theoretical studies 2030 . 32 . Based on these works and our results presented in Fig. 2. we can make some assessments on the differences between Pd and Ir surfaces upon hydrogen sorption. A common feature is that subsurface sorption is by far less stable than in the case of Pd, which in our case can be seen by the sign change between subsurface sorption in Pd (negative) and Ir (positive). The high values of energies at subsurface sites can be related to the absence of hydride formation in bulk Ir, conversely to bulk Pd materials. There are also some differences between $\mathrm{H}$ chemisorption on the Pd and Ir surfaces. The main ones are the competition between hollow and bridge site on $\operatorname{Ir}(100)$ slightly favoring bridge sites. Concerning the $\operatorname{Ir}(111)$ surfaces the previous studies $20,30,32$ have a common finding that on-top position is the most favorable position for hydrogen adsorption. In the present study, within SIESTA calculations, it is indeed found that with regards to $\mathrm{Pd}$, on-top position is more stable and adsorption energy has a negative value getting much closer to the other positions from an energy point of view. However,in our results, there is no change in the sequence of favored surface sites adsorption with regards to Pd with: $E_{f c c}<E_{\text {hcp }}<E_{\text {bridge }}<E_{t o p}$. Going to mixed systems, constituted by a Pd overlayer onto (100) and (111) Ir slabs, one can deduce how the previous properties are combined. The presence of Ir under Pd leads to a moderate weakening of the adsorption onto Pd (see Fig. 2). In return, because of the underneath slab of Ir, sorption at subsurface sites is strongly weakened. In other words, the Pd on top of Ir dictates the adsorption geometry and strength of $\mathrm{H}$ chemisorption, the latter being however somewhat weakened. Moreover, subsurface Ir leads to destabilization of $\mathrm{H}$ absorption. 
Finally, the process of $\mathrm{H}$ penetration (i.e. diffusion to the subsurface) can be inferred in a simple approach by determining the potential energy curve for a displacement of $\mathrm{H}$ perpendicularly to the surface while embedding it progressively in the subsurface region starting from the stable surface hollow sites. In this procedure, the $\mathrm{z}$ coordinate of the penetrating $\mathrm{H}$ atom is fixed and the system is left free to relax in the $(x, y)$ plane. The results, are reported in Table 1 , together with some values of the literature for pure Pd (in the same hydrogen coverage conditions). It is worth noticing that most of the values found in the literature are for the (111) surface of Pd.

Going from pure Pd to Pd/Ir, the resulting activation energies increase. Even if the increase is not drastic, it supports the hypothesis of a destabilization of $\mathrm{H}$ penetration in $\mathrm{Pd}$ subsurface when Ir is used as an underneath slab.

\subsection{Detailed bonding characteristics}

Many adsorption studies are limited to the energetics stage, although an actual control of the reactivity of such material requires a comprehensive study at the electronic structure level to identify the origin of changes in the bonding properties. To this aim, we have extracted from the calculations the projected densities of states (PDOS) at particular sites and orbitals of pure surfaces, mixed ones and finally for the systems with chemisorbed hydrogen. Then we have also extracted the Crystal Orbital Overlap Population (COOP) curves which are a representation of the densities of states $n(E)$ weighted by the overlap population in the wave function at energy $E$ between two atomic orbitals or two atoms ${ }^{14 / 36}$. In this representation, one is able to decompose the interaction in bonding (positive values of the curve), anti-bonding (negative values of the curve) and non-bonding (zero values) regions. The integral of a COOP curve up to the fermi level $\left(E_{f}\right)$ gives then a good insight in the bonding strength evolution of the considered interaction.

It is worth noticing that, in the following, we only consider the case of (100) surfaces but we have verified that the conclusions of this part hold for (111) surfaces.

\subsubsection{Projected densities of states: PDOS}

- Systems before sorption


Fig. 3 Projected density of states (d-PDOS). Left: $\operatorname{Pd}(100)$ and $\mathrm{Pd} / \mathrm{Ir}(100)$ hollow surface sites. Right: Ir subsurface octahedral sites in $\operatorname{lr}(100)$ oriented slab $v s \mathrm{Pd} / \mathrm{lr}(100)$ slab; The vertical lines correspond to the $d$-band center of gravity.
In the analysis of the $d$-PDOS (local $d$ partial density of states) at the Pd and Ir atomic sites of the surfaces of pure Pd and Ir slabs and of Pd covering Ir slabs, the location of the $d$-band center is supposed to give already some indications on the further interaction with adsorbed hydrogen 1937 . Thus, in Fig. 3 are represented the $d$-PDOS for such sites (left) and also for underneath Pd or Ir atomic sites in contact with surface atoms (right). In the latter case, the PDOS at the subsurface site of a pure Ir slab is also represented. The $d$-band center is marked by vertical lines. Going from $\operatorname{Pd}(100)$ to $\mathrm{Pd} / \operatorname{Ir}(100)$ the $d$-band center at the surface is shifted towards lower energies by $0.34 \mathrm{eV}$, which suggests the weakening of further adsorption of hydrogen atoms following the so-called $d$-band position criterion of B. Hammer et al. 37 . Moreover, it is consistent with the energetics study of the preceding section. The width and the detailed structure of the band are only very slightly perturbed and $n\left(E_{F}\right)$ nearly unchanged, indicating a moderate modification of chemisorption properties when covering an $\operatorname{Ir}(100)$ substrate with a Pd monolayer, as compared to pure Pd systems, which is again consistent with the energetics study. The same remark holds for subsurface sorption, going from pure Ir and Ir covered by Pd, with a $d$-band shift even lower $(-0.07 \mathrm{eV})$, which confirms that sorption properties at the interface are dictated by Ir electronic structure.

- H adsorption at (100) surfaces of Pd(100) and Pd/Ir(100)
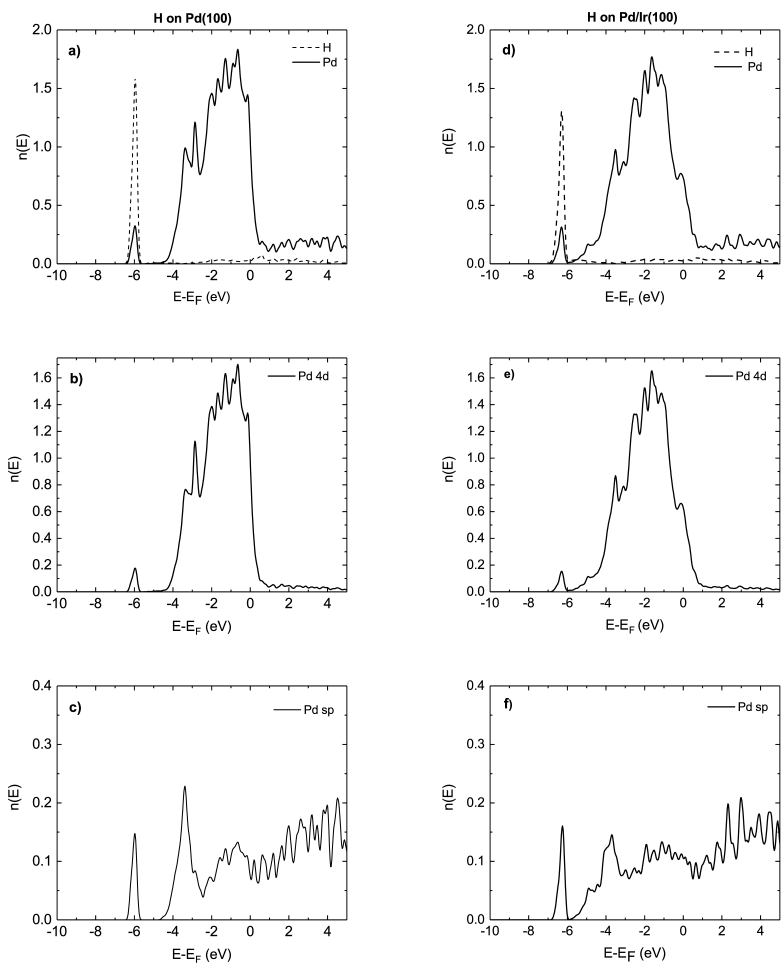

Fig. 4 Projected Densities of States (PDOS) onto Pd and Ir atomic sites in contact with $\mathrm{H}$. $\mathrm{H}$ adsorption at hollow 4-fold (4F) sites on $\mathrm{Pd}(100)$ surface (left) and on $\mathrm{Pd} / \mathrm{Ir}(100)$ surface (right).

Fig. 4 shows the DOS projected onto the $s p, d$-bands of the metallic (100) surface site in contact with $\mathrm{H}$, and to the $\mathrm{H} 1$ s state, in the case of the stable hollow adsorption site. In this 
Table 1 Activation energy $(\mathrm{eV})$ deduced from the potential energy curve of an $\mathrm{H}$ atom penetrating into the subsurface region from hollow sites on Pd.

\begin{tabular}{ccccc}
\hline \hline$\Delta E$ (surf. $\longrightarrow$ subsurf. $)$ & Pd(100) & Pd/Ir(100) & Pd(111) & Pd/Ir(111) \\
\hline Present work & 0.36 & 0.50 & 0.31 & 0.37 \\
Ref. [33] & 0.41 & & 0.40 & \\
Ref. [34] & 0.40 & & & \\
Ref. [24] & 0.32 & & & \\
\hline \hline
\end{tabular}
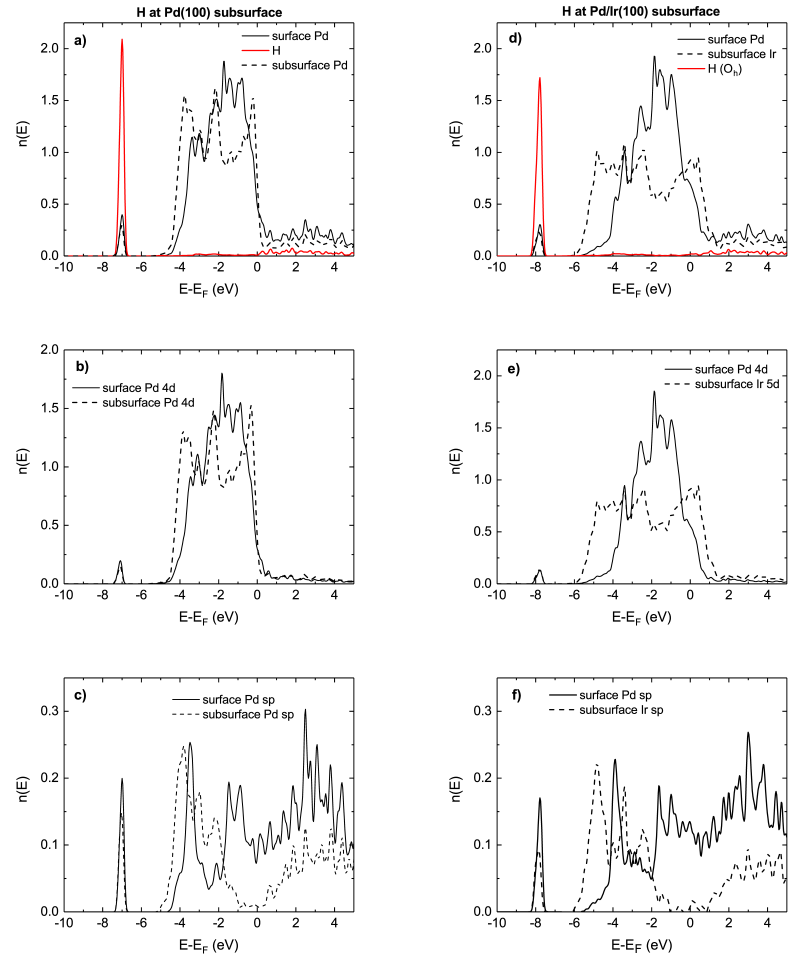

Fig. 5 Projected Densities of States (PDOS) onto subsurface Pd, Ir and $\mathrm{H}$ atomic sites and and their decomposition into $s p$ and $d$ orbital contributions. for $\mathrm{H}$ absorption at subsurface octahedral $\left(O_{h}\right)$ sites. Left: pure Pd system with a $\mathrm{Pd}(100)$ surface. Right: system with a layer of Pd covering an $\operatorname{Ir}(100)$ surface.

configuration, one characteristics is the presence of a peak below the usual $d$-band of the bare Pd surface corresponding to the known split-off state induced by $\mathrm{H}$ adsorption on the metal atoms in contact with $\mathrm{H}$. It corresponds to the bonding overlap of the metal bands with the $\mathrm{H} 1 s$ orbital. Detailed descriptions of this feature and its evolution with the type of sorption site (surface and subsurface) can be found in the literature (see for instance Refs. [14, 19] and references therein). From Fig. 4, it is seen that the $\mathrm{H} 1 s$ orbital is mostly part of the split-off state. Going from hollow sites on $\operatorname{Pd}(100)$ to hollow sites on $\operatorname{Pd} / \operatorname{Ir}(100)$ for $\mathrm{H}$ adsorption (Fig. 4a,d), the split-off state is slightly shifted to lower energies $(\Delta E=-0.5 \mathrm{eV})$. In the decomposition of the surface Pd bands into $d$ and $s p$ contributions (Fig. 4,b,c,e,f) the densities are very similar between Pd(100) and Pd/Ir(100), which indicates that the chemisorption properties towards hydrogen are moderately affected by the presence of an Ir substrate. It can also be seen that the $s p$ contribution to the bonding overlapping between $\mathrm{Pd}$ and $\mathrm{H}$ orbital(s) is significant and should not be completely neglected for quantifying the
metal-H bonding strength.

Fig. 5 shows the DOS projected onto the $s p, d$-bands of the metallic (100) surface and subsurface sites in contact with $\mathrm{H}$, and to the $\mathrm{H} 1 s$ state, in the case of the stable subsurface $\mathrm{O}_{h}$ site. The gap between the $d$-band metallic state and the split-off state is again increased which is due to the increase in coordination 14 . The increase is even larger when the surface Pd layer is supported by an Ir substrate (see Fig. 5]a,d). It is worth mentioning that the width of the gap alone is not a quantity enough relevant to conclude on the hierarchy of the relative stability between the different sites. In particular, any assessment from a simple coordination argument may be misleading. Again here, it can be seen that the $s p$ contribution to the bonding overlapping between $\mathrm{Pd}$ and $\mathrm{H}$ orbital(s) is not negligible (Fig. 5, b,e,c,f).

Finally, it is useful to go beyond the LDOS analysis and introduce the approach of COOP curves, which are more directly related to the bond strength.

\subsubsection{COOP analysis}

- $\mathrm{H}$ adsorbed at stable hollow surface sites

The COOP H-Pd bonding curves (and their integrals) for $\mathrm{H}$ adsorbed at the (100) surface of a pure Pd slab and at the overlayer of Pd supported by an $\operatorname{Ir}(100)$ slab, in the stable hollow fourfold site, are represented in Fig. 6
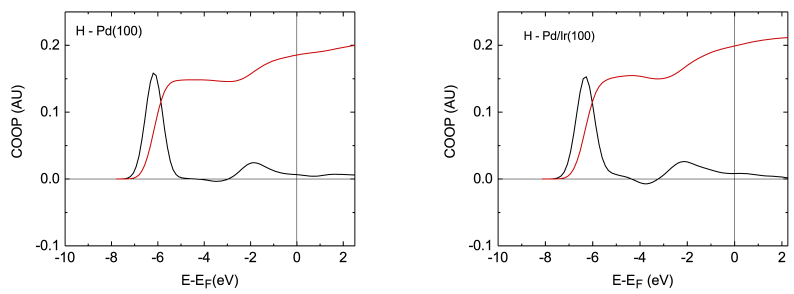

Fig. 6 COOP (black line) and integrated COOP (red line). H-Pd bonding in the case of $\mathrm{H}$ adsorption at surface four-fold hollow sites. Left: pure Pd system with a Pd(100) surface. Right: system with a layer of Pd covering an $\operatorname{Ir}(100)$ surface.

As already mentioned, the split-off state contains most of the bonding effect between metal atoms and $\mathrm{H}$, leading to a first high plateau at low energy in the integrated COOPs. Then after this first plateau, bonding states are still present and contribute to the further increase of the integrated COOP, though to a lower extent. Overall, there is no significant differences between both cases, which shows again that H-Pd bonding at the surface is not strongly affected by the presence of an underneath Ir slab. 

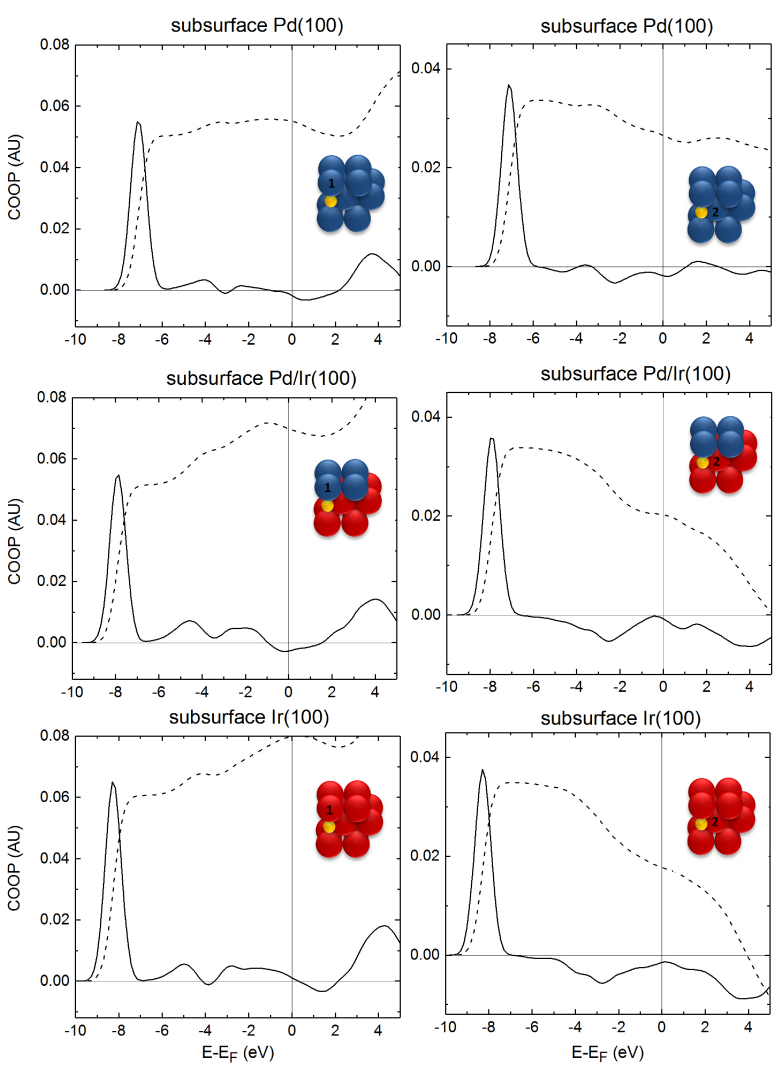

Fig. 7 COOP curves (solid lines) and their integrals (dashed lines) for bonding of $\mathrm{H}$ with $\mathrm{Pd}$ and $\mathrm{Ir}$, the $\mathrm{H}$ atom being in the stable octahedral subsurface position. The left panel is related to the bonding of $\mathrm{H}$ with a surface metal atom and the right one to the bonding of $\mathrm{H}$ with a subsurface metal atom. The ball model in inset shows the considered configuration with red balls for Ir and blue balls for $\mathrm{Pd}$. $\mathrm{H}$ is represented by a small yellow ball. The considered metal atom for the COOP determination is marked with a number: 1 for a surface atom and 2 for underneath atom.

- $\mathrm{H}$ absorbed at stable subsurface sites for an overlayer of Pd on an $\operatorname{Ir}(100)$ slab

The COOP curves and their integrals over energy are represented in Fig. 7. First, in any case the interaction of $\mathrm{H}$ with a surface nearest metallic neighbor leads to a noticeable increase in the integrated curve after the split-off state contribution, independently of the chemical nature of the surrounding atoms. This large increase can therefore be attributed to an electronic surface effect where the bond of $\mathrm{H}$ to a metal surface atom compensates the reduced bonding of the involved surface atom.

Then, in the pure Pd or Ir, $\mathrm{H}$ interaction with the atoms of the subsurface layer, the most important bonding effect still comes from the split-off peak at low energy. In the case of Pd, after the first resulting plateau, the integrated COOP slightly decreases because of small anti-bonding states present in the valence band. These anti-bonding effects are more marked in the case of Ir, leading to a much deeper decrease of the integrated COOP. One consequence is that, at the Fermi level, the value of the orbital overlapping is lower for H-Ir than for H-Pd, which indicates a weaker bonding in the former case, in agreement with the tendency of Pd only to form a hydride in the bulk. The decomposition of the integrated COOP curves into the different orbital contributions shows the most striking difference between Pd and Ir (see Fig. 8). It is related to a strong anti-bonding character in the $1 s-\mathrm{H} / 5 p$-Ir interaction, leading to a non-bonding character of the overall $s p$-Ir interaction with hydrogen, so that going from pure Pd to an environment progressively richer in Ir weakens the overall metal-hydrogen interaction. Conversely, in all pure $\mathrm{Pd}$ systems, the $1 s-\mathrm{H} / s p$-Pd has always an important bonding character, which is of the same order of magnitude as the $1 s-\mathrm{H} / d$-Pd interaction.
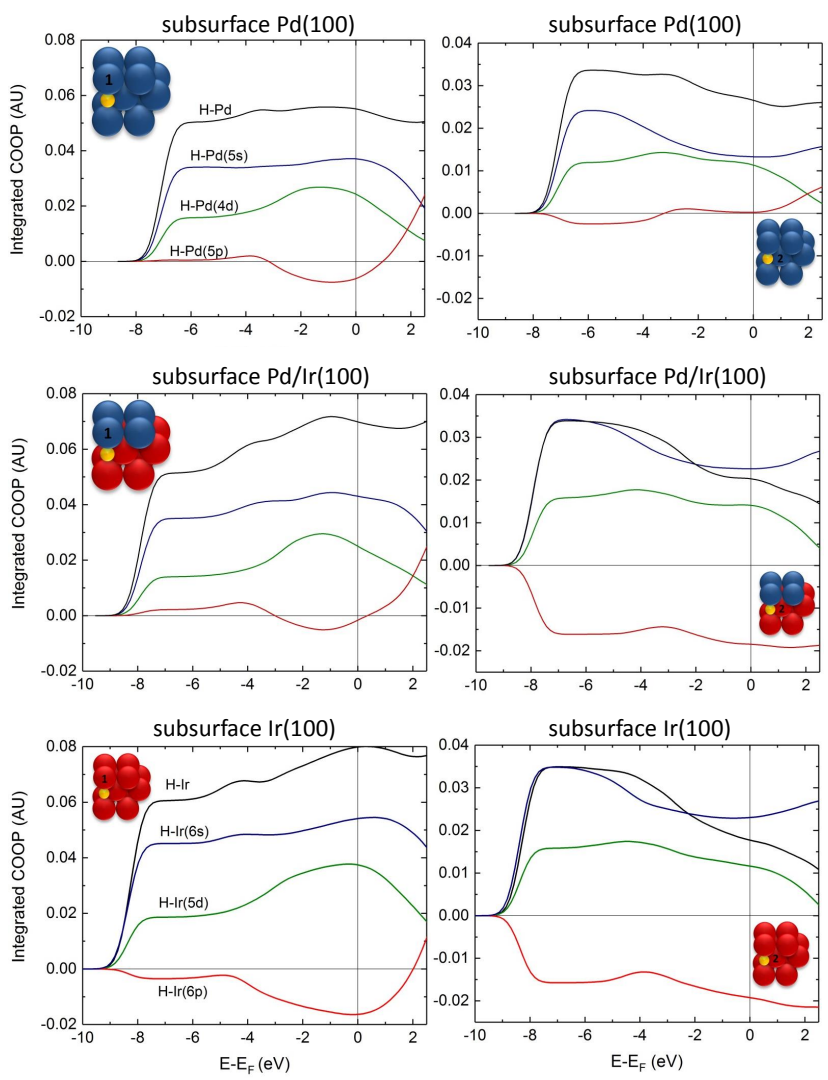

Fig. 8 Integrated COOP curves for bonding of $\mathrm{H}$ with $\mathrm{Pd}$ and $\mathrm{Ir}$, the $\mathrm{H}$ atom being in the stable subsurface octahedral position: decomposition into $s, p$ and $d$ orbitals. The left panel is related to the bonding of $\mathrm{H}$ with a surface metal atom and the right one to the bonding of $\mathrm{H}$ with a subsurface metal atom. The ball model in inset shows the considered configuration with red balls for Ir and blue balls for $\mathrm{Pd}$. $\mathrm{H}$ is represented by a small yellow ball. The considered metal atom for the COOP determination is marked with a number: 1 for a surface atom and 2 for underneath atom.

Finally, let us examine subsurface bonding within the COOP curves taken at the interface of the $\mathrm{Pd}$ overlayer and the Ir substrate in the $\mathrm{Pd} / \operatorname{Ir}(100)$ configuration (Fig. 7). H-(Pd,Ir) curves are rather similar to the case of $\mathrm{H}$-Ir at the subsurface site in the pure Ir slab. In the decomposed integrated curves (Fig. 8), one recognizes the important anti-bonding $1 s$ - $\mathrm{H} / p$-Ir part in the 
valence band at the origin of the deeper decrease in the COOP integral than in Pd, causing the weakening of the $\mathrm{H}$ bonding in presence of Ir.

\section{Conclusion}

Using the strong segregation effect in the PdIr system one is able to retain adsorption properties for surface reactions while preventing hydrogen penetration, which can be detrimental to catalytic performances (as in CO-PROX). As an actual control of the reactivity of such material requires a comprehensive study at the electronic structure level to identify the origin of the changes in $\mathrm{H}$ bonding properties, we have successively determined and examined the PDOS and COOP curves obtained from DFT calculations. The PDOS on atomic sites of bare systems (without $\mathrm{H}$ ) were analyzed according to the so-called $d$-band theory, and corroborate the results of adsorption energetics. The COOP curves show that the main difference between $\mathrm{Pd}$ and Ir with respect to $\mathrm{H}$ sorption relates to a strong anti-bonding character of the $1 s-\mathrm{H} / 5 p$-Ir interaction, leading to a non-bonding character of the $s p$-Ir interaction with hydrogen. Thus, going from pure Pd to an environment progressively richer in Ir increases the anti-bonding contribution, which in turns weakens the overall metal-hydrogen interaction. Conversely,in pure Pd systems, the $1 s-\mathrm{H} / s p$-Pd has always an important bonding character, of the same order of magnitude as $1 s-\mathrm{H} / d$-Pd interaction.

Beyond the PdIr system, the present work shows that an interesting route for designing bimetallic nanocatalysts is to cover a core metal having low affinity with hydrogen (e.g. Ir) with a shell of highly reactive metal (e.g. Pd). The stability of the so-built material is better ensured if the chosen bi-metal has strong surface segregation properties and presents a miscibility gap in the bulk. The theoretical determination of electronic structure features (including $d$ and $s p$ states of the metals) provides a further complementary tool to quantify the evolution of the relative bond strength in the material under gas exposure.

\section{Acknowledgments}

This work was performed thanks to the french Institut du Développement et des Ressources en Informatique Scientifique through a grant of computer time (Project No. 100525).

\section{References}

1 R. Ferrando, Structure and Properties of Nanoalloys, vol. 10, Elsevier (2016).

2 L. Piccolo, Surface studies of catalysis by metals: nanosize and alloying effects, in Nanoalloys: Synthesis, Structure and Properties, D. Alloyeau, C. Mottet and C. Ricolleau, (Eds), Springer London (2012).

3 M. V. Lebedeva, V. Pierron-Bohnes, C. Goyhenex C., V. Papaefthimiou, S. Zafeiratos, R. R. Nazmudinov, V. Costa V., M. Acosta, L. Zosiak, R. Kozubski, D. Muller and E. R. Savinova, Electrochim. Acta, 2013, 108, 605-616.

4 0. Pozdnyakova, D. Teschner, A. Wootsch, J. Kröhnert, B. Steinhauer, H. Sauer, L. Toth, F. C. Jentoft, A. Knop-Gericke, Z. Paál Z. and R.Schlögl, J. Catal., 2006, 237, 17-28.
5 F. Morfin, S. Nassreddine, J. L. Rousset and L. Piccolo, ACS Catal., 2012, 2, 2161-2168.

6 C. Zlotea, F. Morfin, T. S. Nguyen, N. T. Nguyen, J. Nelayah, C. Ricolleau, M. Latroche and L. Piccolo, Nanoscale, 2014, 6, 9955-9959.

7 H. Kobayashi, K. Kusada and H. Kitagawa, Acc. Chem. Res., 2015, 48, 1551-1559.

8 T. Yayama, T. Ishimoto and M. Koyama, J. Alloys Comp., 2015, 653, 444-452.

9 K. Fujii, N. Ishimatsu, H. Maruyama, T. Shishidou, S. Hayakawa S. and N. Kawamura, Phys. Rev. B, 2017, 95, 024116.

10 J. B. A. Davis, R. L. Johnston, L. Rubinovitch and M. Polak J. Chem. Phys., 2015, 141, 224307.

11 T. H. Andriamiharintsoa, A. Rakotomahevitra, L. Piccolo and C. Goyhenex, J. Nanopart. Res., 2015, 17, 217.

12 J. B. A. Davis, S. L. Horswell, L. Piccolo and R. L. J. Johnston, J. Organomet Chem., 2015, 792, 190-193.

13 T-E. Fan, I. Demiroglu, H. A. Hussein, T. Liu and R. L. Johston, Phys. Chem. Chem. Phys., 2017, DOI:10.1039/C7CP04811D.

14 J. Paul and P. Sautet, Phys. Rev. B, 1996, 53, 8015-8027.

15 W. Dong, V. Letendu, P. Sautet, A. Eichler and J. Hafner, Surf. Sci., 1998, 411, 121-136.

16 O. M. Lovvik and R. Olsen, Phys. Rev. B, 1998, 58, 10890-10898.

17 X. Q. Qi, Z. D. Wei, L. Li, M. B. Ji, L. L. Li, Q. Zhang, M. R. Xia, S. G. Chen and L. I. Yang, Computational and Theoretical Chemistry, 2012, 979, 96-101.

18 C. Zhang and A. Michaelides, Surf. Sci., 2011,605, 689-694.

19 V. Pallassana, M. Neurock, L. B. Hansen and J. K. Norskov Phys. Rev B 1999, 60, 6146-6154.

20 J. Greeley and M. Mavrikakis J. Phys. Chem. B 2005, 109, 3460-3471.

21 O. M. Lovvik and R. Olsen, J. Chem. Phys., 2003, 118, 3268-3276.

22 A. Lebon, A. García-Fuente, A. Vega and F. Aguilera Granja, J. Phys. Chem. C, 2012, 116, 126.

23 F. Juarez, G. Soldano, E. Santos, H. Guesmi, F. Tielens and T. Mineva, Compu tation, 2016, 4, 26.

24 P. M. Quaino, R. Natzmutdinov, L. F. Peiretti and S. Santos, Phys. Chem. Chem. Phys., 2016, 18, 3659.

25 J. M. Soler, E. Artacho, J. D. Gale, A. Garcia, J. Junquera, P. Ordejon and D. Sanchez-Portal, J. Phys. Condens. Matter, 2002, 14, 2745-2779.

26 N. Troullier and J. L. Martins, Phys. Rev. B, 1991, 43, 1993-2001.

27 L. Kleinman and D. M. Bylander, Phys. Rev. Lett., 1982, 48, 1425-1428.

28 Garcia A.; ATOM User Manual, https://departments.icmab.es/leem/siesta/ zcam14/Manuals/atom.pdf

29 I. López-Corral, E. Germán, A. Juan, M. A. Volpe and G. P. Brizuela, International Journal of Hydrogen Energy, 2012, 37, 6653-6665.

30 W. P. Krekelberg, J. Greeley, M. Mavrikakis, J. Phys. Chem. B, 2004, 108, $987-$ 994.

31 L. Kristinsdóttir and E. Skúlason, Surf. Sci., 2012, 606, 1400-1404.

32 D. Vasić, Z. Ristanović, I. Pasti and S. , Russian Journal of Physical Chemistry A, 2011, 85, 2373-2379

33 P. Ferrin, S. Kandoi, A. U. Nilekar and M. Mavrikakis, Surf. Sci., 2012, 606 679-689.

34 R. A. Olsen, P. H. T. Philipsen, E. J. Baerends, G. J. Kroes and O. M. Lovvik, J. Chem. Phys., 1997, 106, 9286.

35 R. A. Olsen, G. J. Kroes, O. M. Lovvik and E. J. Baerends, J. Chem. Phys., 1997, 107, 10652.

36 R. Dronskowski, Computational Chemistry of Solid State Materials, Wiley-VCH, 2005.

37 B. Hammer and J. K. Norskov, J. K. Eds R. M. Lambert and G. Pacchioni, Chap. Chemisorption reactivity on supported clusters and thin films, 1997, 331-351. 